\title{
On the use of thermal inertia in building stock to leverage decentralised demand side frequency regulation services
}

\author{
Sean Williams*, Michael Short and Tracey Crosbie
}

Technology Futures Institute, Teesside University, TS1 3BX, UK

*Corresponding author: E-mail: sean.williams@tees.ac.uk; Tel: +44 (0)1642 342528

\begin{abstract}
Most governments are applying financial instruments and other polices to encourage distributed renewable electricity generation (DREG). DREG is less predictable and more volatile than traditional forms of energy generation. Closure of larger fossil-fuelled power plants and rising share of DREG is reducing system inertia on energy networks such that new methods of demand response are required. Usually participation in non-dynamic frequency response is reactive, affecting the duty cycle of thermostatically controlled loads. However, this can adversely affect building thermal efficiency. The research presented takes a proactive approach to demand response employing heat transfer dynamics. Here, thermal dynamics exhibit a significantly larger inertia than electrical power consumption. Thus, short-term fluctuations in energy use should have less effect on temperature regulation and user comfort in buildings than existing balancing services. A prototype frequency sensor and control unit for proactive demand response in building stock is developed. The paper reports on hardware-in-the-loop simulations, testing real thermal loads within a simulated power network. The instrumented approach adopted enables accurate real-time electrical frequency measurement, while the control method offers effective demand response, which suggest the feasibility of using decentralised frequency control regulation as a novel approach to existing demand response mechanisms.
\end{abstract}

Keywords Frequency regulation; decentralised control; demand response.

\section{Nomenclature}

\begin{tabular}{|ll|}
\hline$d$ & transport delay, $\mathrm{s}$ \\
$D$ & load damping constant, $\mathrm{s}$ \\
$\Delta f$ & frequency deviation, $\mathrm{Hz}$ \\
$H$ & inertia time constant, $\mathrm{s}$ \\
$I$ & TCL controller integral gain \\
$K_{i}$ & ALFC secondary loop gain, p.u. MW/Hz s \\
$K_{h}$ & thermal load gain \\
$P$ & TCL controller proportional gain \\
$\Delta P_{d}$ & step change in power demand, p.u. \\
$\Delta P_{g}$ & change in hydraulic amplifier output \\
$\Delta P_{h p}$ & electrical power deviation, p.u. \\
$\Delta P_{t}$ & change in turbine power output \\
$R$ & regulator, Hz/p.u. MW \\
$\Delta T$ & temperature deviation, p.u. \\
$T_{g}$ & governor time constant, $\mathrm{s}$ \\
$T_{h}$ & thermal load time constant, $\mathrm{s}$ \\
$T_{t}$ & turbine time constant, s \\
$\bar{x}$ & sample mean \\
$n$ & number of entries \\
$S$ & sample standard deviation \\
$\sigma$ & standard error \\
$Z$ & standardized test statistic \\
\hline
\end{tabular}

\section{Introduction}

Historically national electrification followed a trajectory of increasing integration and centralisation [1]. In centralised electricity networks the transmission system operators (TSOs) are primarily responsible for balancing the network [2]. Recently however an expanding share of power generation on the distribution network is challenging existing approaches to balancing supply and demand [3,4]. In light of this, it is widely argued that distributed system operators (DSOs) need to take on some of the responsibility for balancing electricity networks [1]. Improving coordination between TSO-DSO to unlock new flexible services that seek to optimise the distribution grid operations will encourage more active participation of the consumer in demand side response DSR [5]. However, as outdated perceptions of technology cost and performance continue, the centralised approach to energy production, delivery and consumption fails to cope with an evolving energy landscape [6-8].

Most conventional generation plants use large synchronous machines to meet time-invariant imbalances between supply and demand. This stored kinetic energy provides rotational inertia that helps absorb extemporal transient 
imbalances. However, a downward trend in traditional centrally-dispatched fossil-fuel generation plants and a rising share of DREG, with at best limited inertial response, increases the uncertainties of grid stability particularly during extreme stress events [9]. In the absence of any meaningful alternative to compensate for low system inertia, e.g. energy storage systems, the likelihood of frequency falling below the limits that trigger an unwanted disconnection of services is exacerbated $[10,11]$.

To cope with frequency excursions in power networks, and potential stability concerns, network operators continue to encourage participation in DSR. However, an increasing demand due to the introduction of electric vehicles and electrification of heat poses a real threat how network operators will manage what is largely a passive network [12]. It seems necessary to formulate a new classification of DSR in the distribution grid [13]. This paper proposes a decentralised proactive approach to balancing and system optimisation that will assure the longevity of existing infrastructure and safeguard against future load demands on the network. The performance of proactive frequency control is assessed with a set of computer models and hardware-in-the-loop simulations, which mimic the working conditions of a thermal load.

\subsection{State-of-the-art}

The existing literature on DSR is rich and heterogeneous. Grid operators in the UK have typically targeted energyintensive industries to participate in flexible regulatory services by modulating their power consumption behaviour within an agreed contractual framework [5]. Yet, a growing share of DREG is leading researchers to seek a new classification of flexible approaches to balancing the network [14]. For example, in an article that analyses the balancing of supply and demand in domestic energy grids, researchers conclude that providing a range of flexible options appears to stimulate household consumers behaviour how they engage with energy [15]. However, the motivations offered to effect useful power reduction for many small or medium sized consumers on the network are not as persuasive and the commercial viability of participating in such DR schemes is not prevalent [16]. Electricity interconnectors can help keep European grids secure by making it easier to manage stress events caused by power plant failures or intermittent DREG [17]. But, they too require significant investment and depend on the ability of partnered countries grid infrastructures to manage bi-directional energy flows. In addition to interconnectors, energy storage has a role in future energy markets [18]. Here, energy storage could contribute to solving the problem of stress events and seasonal imbalances between supply and demand, but storing electricity is expensive and technically challenging [19]. Furthermore, shortfalls of current battery storage technologies mean they are not able to cope with the variability of generation and demand imbalances quickly enough to maximise economic benefit [20]. To overcome some of these constraints there is a growing interest in supercapacitors and electrochemical batteries (hybrid energy storage system) for grid supporting applications [21]. But, the market is waiting for improvements in volumetric capacity of energy storage and it is unlikely that these improvements will materialise in the required timeframe [22]. Therefore, alternative mid to long-term solutions that contribute to the stability of the grid are required.

Alternative strategies that provide reactive automatic frequency control through interruption of demand are available [23]. However, to actively participate in any demand-side initiative, the outcomes are largely dependent on the availability and reliability of a communication network. Several papers use a centralised optimisation-based approach to modulate the behaviour of thermostatically controlled loads, such as refrigerators, heating ventilation and air conditioning (HVAC) systems; which allow the controller to affect the duty cycle of the appliance [24,25]. Household appliances are an attractive source for participation in electricity markets by providing control reserves. The thermal characteristics of such appliances been more effective when considered as a population rather than individual assets [26]. By centrally controlling the duty cycle of a distributed population of individual appliances the role of an aggregator has the potential for greater impact on the energy network [27]. A study that validated a centralised approach to primary frequency control of electric vehicles with a centralised communication architecture found that communication delay was not an obstacle to the control strategy adopted [28]. However, in the context of DR, with limited service, consumers' ability to give up control of in-use loads is compromised; even in cases of full service provision, latency issues rule out (in almost all cases) the leverage of demand-side responses in the very short $(<15 \mathrm{~s})$ timescales required for primary frequency control [29]. In the absence of service, their actions might even aggravate the situation further by increasing their overall energy consumption at a time when a balancing controller is attempting to restore frequency equilibrium through load reduction. Moreover, when aggregated populations of TCLs where control does not exclusively rely on stochastic switching then a synchronisation phenomenon can lead to unsatisfactory behaviour [30,31]. Apart from undesirable frequency control activation TCLs can advance further instability and system failure. Nevertheless, the utility of TCLs continues to be the focus of research when analysing the potential contribution for short-term modulation in decentralised control flexible demand response scenarios [23,32-34].

\subsection{Novelty}

There is a tangible link between economic growth and increased demand in energy for space heating and air conditioning [35]. Therefore, thermal storage in buildings is a growing resource. According to recent studies, energy consumed in residential and commercial buildings accounts for $20.1 \%$ of the globally delivered energy in 2016 [36]. 
Building efficiency strategies can be: (i) passive, i.e. seeking to improve the fabric of buildings, and (ii) active, i.e. encompassing improvements to space heating by decreasing the energy demand of the building [37,38]. Balancing services provided by manipulating the load profile of domestic appliances, or exploiting the amount of thermal mass in a building has received some attention $[39,40]$. However, the literature on provision of similar gains through decentralised proactive frequency control and optimisation of indoor comfort temperatures in commercial building stock by exploiting properties that contribute to thermal stability is less apparent [41].

Differences between previous approaches to DR and the one presented in this paper are twofold: (i) the presented approach is not dependent upon a national ICT infrastructure (decentralised control) and (ii) the approach avoids discontinuous (on/off) switching of loads, thus, avoiding synchronisation and restoration loading issues. The approach discussed here aims to replicate the primary 'droop' control present on supply generators on the demandside, exploiting electro-thermal couplings. It can operate independently at the building or block of buildings scale, in either islanded mode or on-grid. It controls space heating, without compromising occupancy comfort, using a signal set to change proportionally to mains frequency, thus avoiding long-term oscillatory behaviour. The findings presented demonstrate this research offers a simple and viable alternative to other market mechanisms, such as fast acting frequency response services.

As the energy landscape evolves, the traditional centralised energy control and production topology is in transition towards one that encourages more devolved, decentralised generation and distribution [42]. The number of heat pumps sold in the European Union from 2010 to 2015 has risen to 800,000 [43]. This upsurge in heat pumps on the market is set to continue. Studies have identified heat pumps as the most energy-efficient and cost-effective system that comply with EU Directive on the Energy Performance of Buildings [44]. A recent article that evaluates the potential gains of heat pumps in domestic buildings observes one of the main motivators for households to switch from natural gas boilers to heat pumps is based on economic reward [45]. High performance values under different load conditions make heat pumps ideal candidates for the type of proactive decentralised frequency control and regulation proposed in this work. In the context of smart grids, capacity control of heat pumps by regulating shortterm frequency excursions in electricity demand as an integrated component of a localised building energy management (BEM) system, may provide a low-cost and resilient mid-term solution to help secure grid stability [46].

\subsection{Contributions and structure}

Although provision of primary frequency control has been previously identified in literature the drawback when operating in the context of demand response is its dependency on a robust communication system. Aggregation of TCLs presents unique challenges as a reactive control strategy attempts to minimise the synchronisation phenomenon, usually by dispatching some form of elaborate stochastic switching mechanism. With respect to existing literature, the contribution of this work proposes a proactive decentralised approach to demand response while preserving the characteristics necessary for primary frequency control.

The slow transient behaviour of thermal mass of a building allows for continuous dynamic control proportional to a change in grid frequency without affecting the temperature in the immediate surrounding. As a general tendency it can be concluded that for most buildings this will improve building energy efficiency without compromising occupancy thermal comfort, at the same time as providing a positive effect on the grid reserve generation capacity margin.

An additional contribution of this work is an analysis of a prototype low-cost frequency measurement unit that can be deployed without the reliance of centralised communication for accurate frequency measurement. An analysis of measured frequency and data obtained from transmission system operators makes the comparison between different solutions and methodologies more credible. This opens the possibility of consumer participation in DR at reduced cost.

The remainder of this paper is organised as follows: Section 2 describes the configuration of software and hardware components used in the hardware-in-the-loop (HIL) real-time simulation tests to demonstrate decentralised primary frequency control. Section 3 then presents the simulation results. Finally, Section 4 presents the conclusions drawn from the results and offers some concluding remarks.

\section{$2 \quad$ Materials and Methods}

\subsection{Proposed method}

Decentralised demand-side frequency regulation when used in building stock can regulate short-term frequency excursions in demanded electrical energy [47]. The proposed decentralised demand response method can operate with no national communication network but requires access to a reliable source of mains frequency measurement. Figure 1 illustrates the general approach. Here, when a power disturbance $(\Delta P d)$ is applied to a single area power system driven by a lumped parameter non-reheat steam turbine, the decentralised frequency control (DFC) regulation action can have a positive influence on reserve generation capacity by arresting the measured frequency excursion in real-time. Moreover, a small variance in building (zonal) temperature setpoint will have negligible effect on user comfort. 


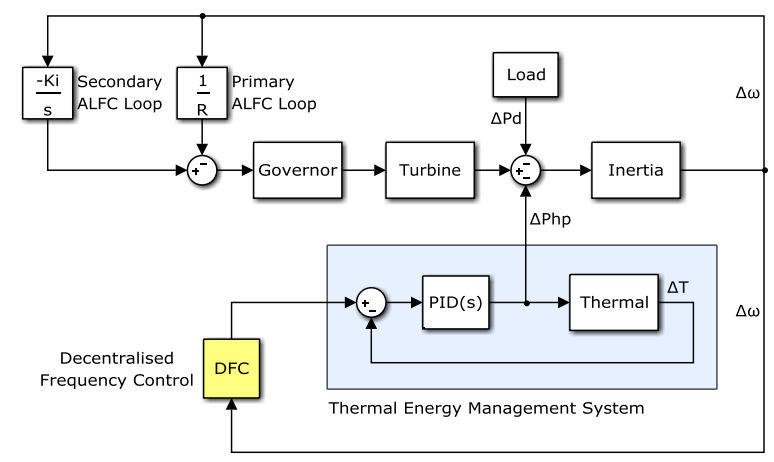

Figure 1 Proposed role for DFC-Primary regulation

\subsection{Frequency measurement}

On a synchronous generator, the frequency of the interconnected power system is implicitly instrumented via the generator tachometer, which measures shaft speed locally. At a load site, however, a dedicated means is required for frequency measurement, unless real-time communication is employed. But, the reliance on advanced communication networks to convey this information at sufficient resolution and maintain real-time accuracy at decentralised nodes is problematic $[48,49]$. Many examples of frequency measurement estimation algorithms and techniques are available in the literature (e.g. [50]). However, in practice the implementation of decentralised frequency regulation is not always favourable and quite often cost-inhibitive. This research offers a low-cost working prototype using the zero crossing detection technique, constructed using an Arduino Mega 2560 - ATmega2560 low-power CMOS 8-bit microcontroller [51]; designed to measure and visualize real-time mains frequency at a resolution of $100 \mathrm{mHz}$ at least once every second. Figure 2 illustrates a breakout view of the signal conditioning and frequency measurement modules.

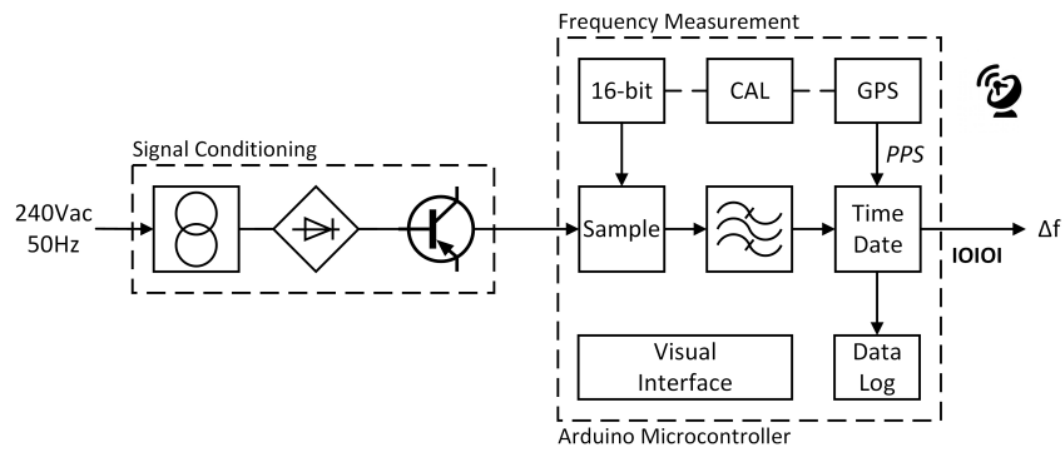

Figure 2 Frequency sensor breakout

The design includes a GPS shield providing a time-base that is synchronised to coordinated universal time (UTC). In addition to geographical positional information, a GPS 1 pulse per second digital signal allows highly accurate time date stamping for data collection, along with self-calibration of the device when a GPS fix is established. The latter is necessary to maintain measurement accuracy despite imperfections and temperature sensitivity of the microcontroller crystal oscillator. A local communication option in the form of a TIA-232 serial data interface allows the device output to be injected into a BEM system. An internal microSD card, or external memory device can be used to record and store information for subsequent analysis. Real-time readout of frequency measurement and visual warnings are provided using a 16x2 matrix LCD display and series of LED.

After construction, inferential statistics was used to test the hypothesis that the Arduino frequency sensor data is as good as data accessible from the National Grid. Using two-sample f-test for variance and two-sample z-test for difference between two means, given a sample number of 239, the variance $s_{n}^{2}$ and standard deviation $s_{n}$ were calculated, Eq. (1) and Eq. (2).

$$
\begin{gathered}
\bar{x}=\frac{\sum x}{n} \\
s=\sqrt{\frac{\sum(x-\bar{x})^{2}}{n-1}}
\end{gathered}
$$

Using the same data sample, a standard error and standardised test statistic were calculated using Eq. (3) and Eq. (4). 


$$
\begin{gathered}
\sigma_{\bar{x}_{1}-\bar{x}_{2}}=\sqrt{\frac{s_{1}^{2}}{n_{1}}+\frac{s_{2}^{2}}{n_{2}}} \\
z=\frac{\left(\bar{x}_{1}-\bar{x}_{2}\right)-\left(\mu_{1}-\mu_{2}\right)}{\sigma_{\bar{x}_{1}-\bar{x}_{2}}}
\end{gathered}
$$

\subsection{Software simulation of decentralised frequency control regulation}

A simulation model to determine the mains frequency response of a simplified linear power system model was created using MATLAB/Simulink $@$ software [52]; sample rate $T s=0.02$. For illustrative purposes, a single area system driven by a lumped parameter non-reheat steam turbine was implemented [53]. Initially, the function of automatic load frequency control (ALFC) primary and subsequently secondary loops that control the prime mover and generator outputs were simulated to provide a baseline for comparison.

In order to propose a role for primary regulation on the demand (load) side, some assumptions are first made: (i) the control signal from the regulator is employed to actuate a TCL which either heats or cools the area under consideration, and (ii) in the context of this analysis, the control mechanism excludes any supplementary heat source to compensate for deviations of measured temperature that might otherwise compromise the occupancy comfort. Detailed models of TCL and building thermal behaviour can be found in literature (e.g. [37,54]). They are usually based on physical principles of mass, energy and momentum transfer and consist of complex partial differential equations that capture the building thermal and physical characteristics. However, in practice simplified first-order models can perform just as well as more complicated models [55]. Here, the dynamic relationship between the electrical power delivered to the electro-thermal converter $P_{h p}(t)$ and the temperature of the heated zone $T(t)$, can be very well approximated by a First-Order Plus Dead Time (FOPDT) transfer function (t.f.) describing deviations away from a nominal input/output steady-state operating point. Using per-unit representations for $P_{h p}(t)$ and $T(t)$ to eliminate the steady-state gain in the model, and using $\Delta$ to represent deviations, the transfer function for the thermal response becomes:

$$
G(s)=\frac{\Delta T(s)}{\Delta P_{h p}(s)}=\frac{e^{-s d}}{1+\tau s}
$$

Where $d$ represents the model dead-time and $\tau$ the time constant. The time constant can be assumed to be in the range of 10-30 minutes and the dead-time between 0-5 minutes for a typical building [10]. A PT326 Process Trainer models common industrial situations in which temperature control is required [56]. Using this hardware to emulate building thermal behaviour, process dead time $(d)$ and process time constant $(\tau)$ measurements recorded during an open-loop step test were used to implement a proportional-integral (PI) controller following a Lambda tuning methodology [57]. This form of internal model control (IMC) [58] completes a setpoint change in about $4 \lambda$ seconds when operating in closed-loop mode, without overshoot, where $\lambda=\left(t_{s}-d\right) / 4.6$, settling time $t_{s}=50$, and $d$ is the TCL (PT326 Process Trainer) calculated transport delay.

The contribution of the proposed DFC-Primary regulator is possible using this simplified thermodynamic model of a building thermal control system. To characterise the extent of a simulated secondary demand-side response in a system complete with a DFC-Primary regulator, an idealised secondary demand response command event (network latency is assumed to be zero) was introduced during further tests.

Having established a computer model of a simplified linear power system and building thermal control system, a contingency load was introduced when a balance in supply and demand was at equilibrium, the frequency was at a nominal $50 \mathrm{~Hz}$ and the steady state frequency error was zero. In all cases, simulations were carried out using time constants and other parameters taken from representative sources (Appendix 1) under the assumption that a large step-change in power disturbance $(\triangle P d)$ occurred at the beginning of each simulation. In the model illustrated in Figure 3, a power system rating of $300 \mathrm{MVA}$ is assumed. A contingency of $75 \mathrm{MW}$ power disturbance $(\triangle P d=$ $75 \mathrm{MW}, 0.25$ p.u.) step response at $t=750 \mathrm{~s}$ was introduced, allowing time for the system to initialise, before triggering a change in frequency. Simulation results are presented in Section 3. 


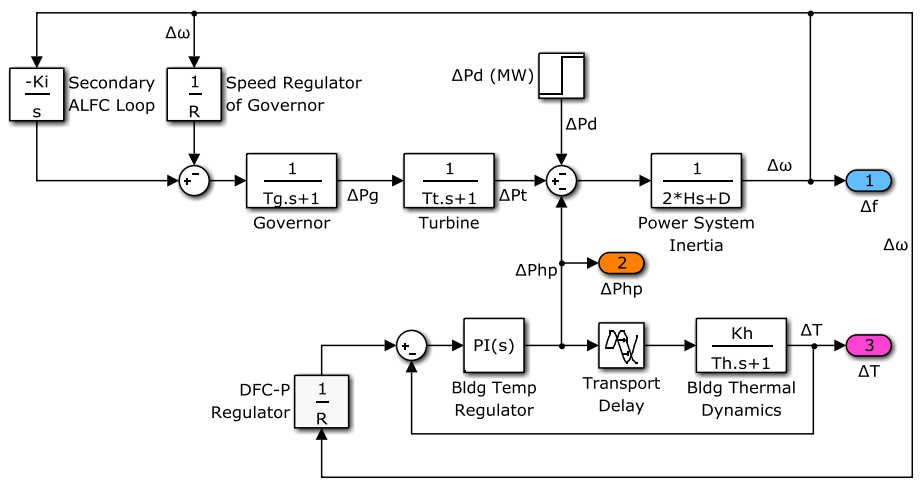

Figure 3 Simulink model of decentralised primary frequency control

\subsection{Hardware in the loop with thermostatically controlled load}

Next, HIL simulations were conducted, initially replacing the FOPDT t.f. (simulating the dynamic relationship between the electrical power delivered to the electro-thermal converter and the temperature of the building) with a PT326 Process Trainer (Appendix 2). A PCI-DAS6014 hardware item, configured to provide an interface between the on-bench TCL and PI controller, was implemented in MATLAB/Simulink@ (Figure 4). The PT326 Process Trainer mass flow of air temperature was measured using a thermistor placed at a position such that the spatial separation between the heater coil and thermistor introduced a transport delay $d=0.45$ into the system. In practice this time delay is much greater, typically 5-10 minutes depending on a multitude of factors. A secondary advisory temperature measurement taken from a DHT22 combined temperature and humidity sensor was also positioned directly into the PT326 Process Trainer mass airflow outlet. A $433 \mathrm{MHz}$ RF communication network was established between the remote DHT22 sensor and MATLAB/Simulink@ model to enable the observer to record temperature data during each test cycle.

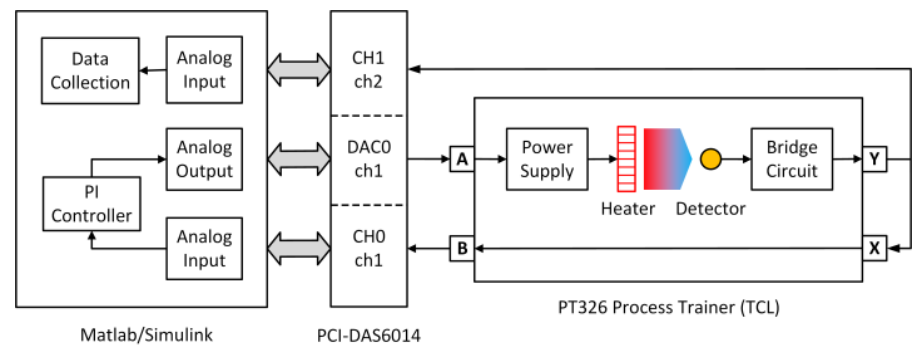

Figure 4 Hardware in the loop test environment

A HIL simulation test to determine the mains frequency and external TCL (PT326 Process Trainer) temperature response was then created using MATLAB/Simulink@ software (Figure 5). After calibration, the PT326 Process Trainer hardware item temperature setpoint (OF1) was set and maintained at 7.5, ensuring a process temperature measured mid-scale on the instrumented temperature gauge; which registered a reading equivalent to $40^{\circ} \mathrm{C}$. $\mathrm{A}$ contingency of $75 \mathrm{MW}$ power disturbance $(\triangle P d=75 \mathrm{MW}, 0.25$ p.u. $)$ step response at $t=750 \mathrm{~s}$ was introduced, allowing time for the system to initialise, before triggering a change in frequency. Plots showing simulated frequency response $\Delta f(t)$, recorded variation in temperature $\Delta T(t)$ and electrical power $\Delta P_{h p}(t)$ delivered to the electrothermal converter obtained from computer simulations tests (Figure 3 ) and HIL-test simulations makes the analysis of decentralised frequency control performance more credible. 


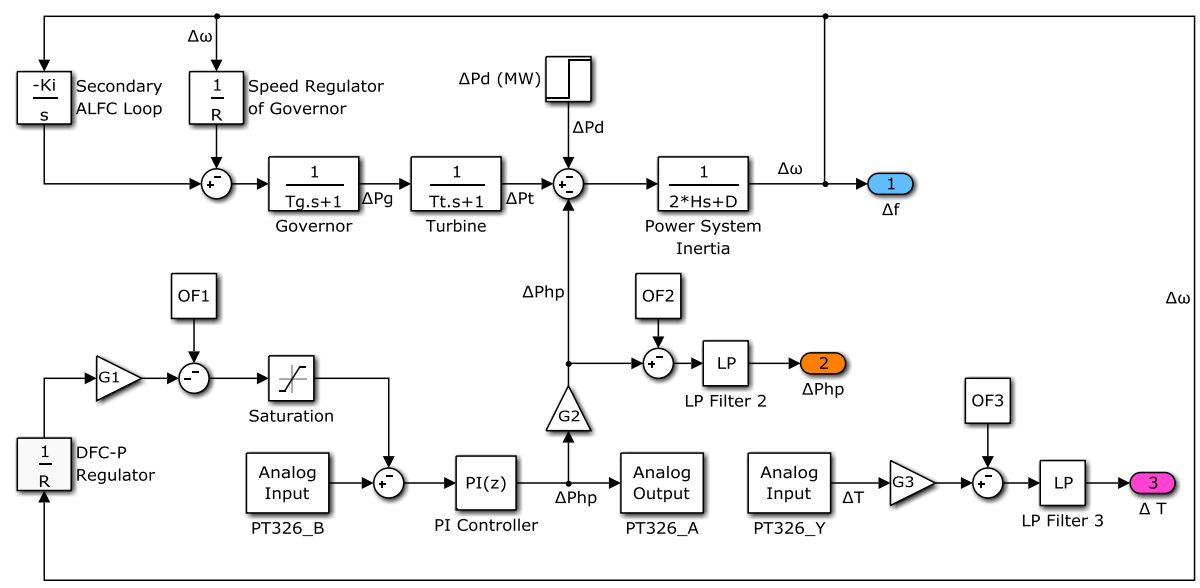

Figure 5 Hardware in the loop frequency regulation

\subsection{Hardware in the loop with prototype mains frequency sensor}

Additional HIL testing injecting a continuous input stream of real-time mains frequency measurement output from the Arduino frequency sensor into a modified computer model was completed. Analysis of the TCL recorded temperature evolution when an input stream of mains frequency data was input into a decentralised frequency control regulator was performed. The results of running an extended model that compared the variance in temperature recorded using the on-bench TCL HIL test (Figure 6) against values recorded from a computer model that substituted the TCL with a FOPDT t.f. are presented in the next section.

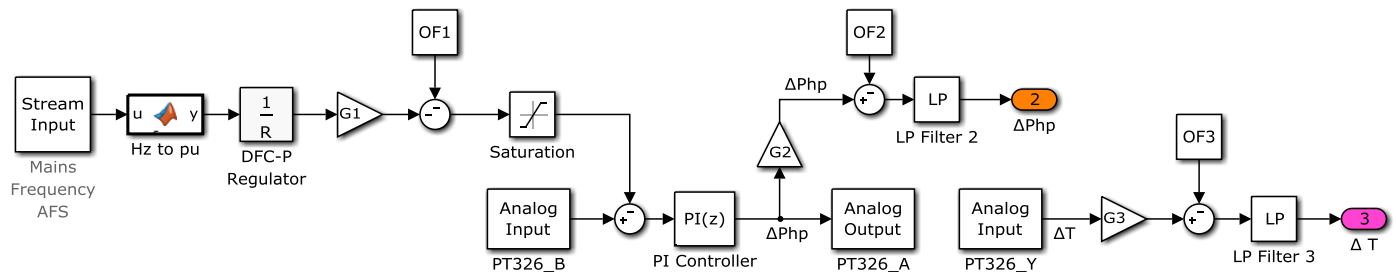

Figure 6 Model of stream input frequency to TCL

\section{Results}

We begin by reviewing the correlations between data recorded using a prototype frequency sensor and data extracted from the balancing mechanism reporting service (BMRS) [59]; (Figure 7) [dataset] [60]. Statistical inference verified the device output performance based on probability. [16]

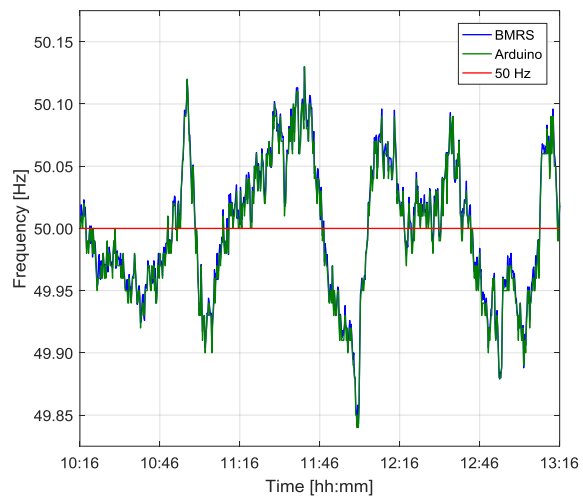

Figure 7 Arduino frequency sensor and BMRS data

15 Dec 2016 10:16 15 s resolution

Tests for homogeneity of variances were applied to compare the normal population using equal size data from two independent samples (frequency sensor data sample and BMRS data sample). Given a population recorded over a 1hour period at a resolution of $15 \mathrm{~s}$, created a sample number $n=239$. Calculating Eq. (1) and Eq. (2) variance, $s_{1}^{2}=$ 0.00059703 , and standard deviation, $s_{2}^{2}=0.00058566$, respectively showed $s_{1}^{2}>s_{2}^{2}$. Hence, $s_{1}^{2}$ and the standard error $\sigma_{1}^{2}$ were used to represent the sample and population variances for the Arduino frequency sensor. Two-sample f-test for variance and two-sample z-test for difference between means were conducted using random sample data 
recorded at $15 \mathrm{~s}$ resolution for a 60-minute period commencing 14:23:00 on 14/12/2016. Results of both tests indicate at the $5 \%$ level of significance that there was no difference in the two independent samples variance and mean frequency data.

Figure 8 reports the results of the action of a small-gain regulator when introduced into the simulation model DFCPrimary loop. Here, not only does the regulator eliminate previously observed frequency oscillations but also reduces the measured frequency deviation. A further test cycle with the ALFC secondary loop included confirmed the action of the small gain integrator slowly restored the frequency deviation to zero over an extended period. The effect of proactive frequency control on maintaining frequency equilibrium during network stress is profound. This positive outcome implies the gap on reserve generation capacity margins is reduced and the time period to restore frequency equilibrium lessened; ( $8 \mathrm{~min}$ compared to $14 \mathrm{~min}$ ). Further analysis compares the time-series data recorded from a simulated model against data recorded from a HIL simulation employing a controllable external TCL (PT326 Process Trainer). In particular, Figure 8(a) [dataset] [60] illustrates the effect on frequency response when the DFCPrimary loop was not present. The transient effect on all three outputs when a secondary demand side event was initiated is also illustrated. Comparing all three outputs it is evident that the computer model is an accurate representation of the equipment used during the HIL tests (comparing PT326 and Simulink plots). Moreover, the simulated secondary demand response event improves the frequency response settling time while retaining the benefits of the DFC-Primary regulator, i.e. reduced initial frequency deviation (49.4 Hz compared to $48.9 \mathrm{~Hz})$ and no frequency oscillatory behaviour. The temperature and electrical power plots illustrate a reduced steady state condition is achieved when a secondary demand response is present, which is determined by the magnitude of the simulated secondary demand response command signal. The small thermal transient in the absence of any secondary demand response, Figure 8(b) [dataset] [60], is likely to have little impact upon building thermal comfort; although this behaviour should be investigated further.

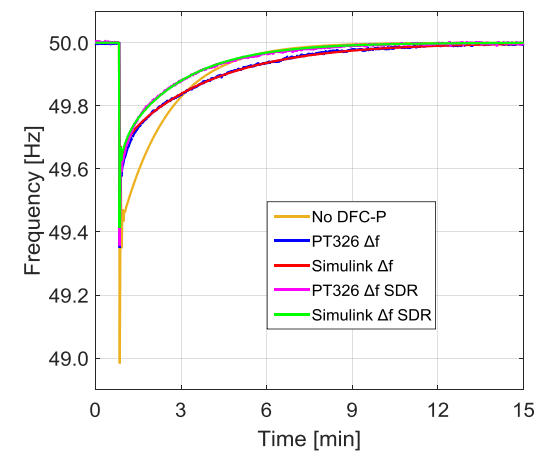

(a)

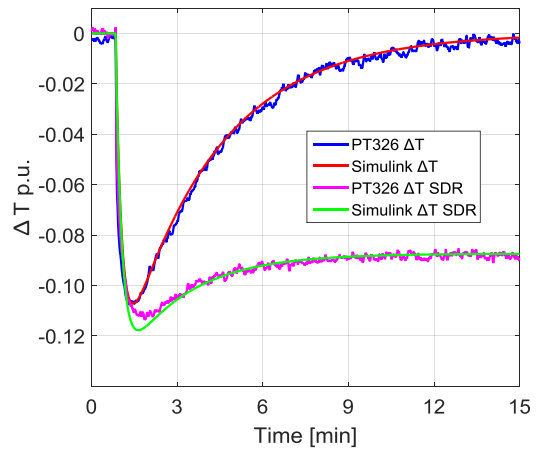

(b)

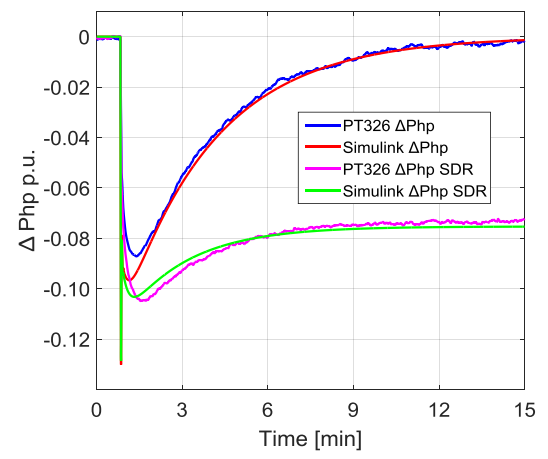

(c)

Figure 8 Decentralised frequency control regulation

(a) frequency response, (b) temperature response, (c) electrical power

Figure 8(c) [dataset] [60] reveals secondary benefits, the contribution of electrical power restoration to the primary energy source, i.e. the single area system. This opposing transient behaviour compensates for any deviation on demand side load within a power system, contributing to a more proactive approach to restoring any detected imbalance in supply and demand side load at the same time as establishing frequency equilibrium.
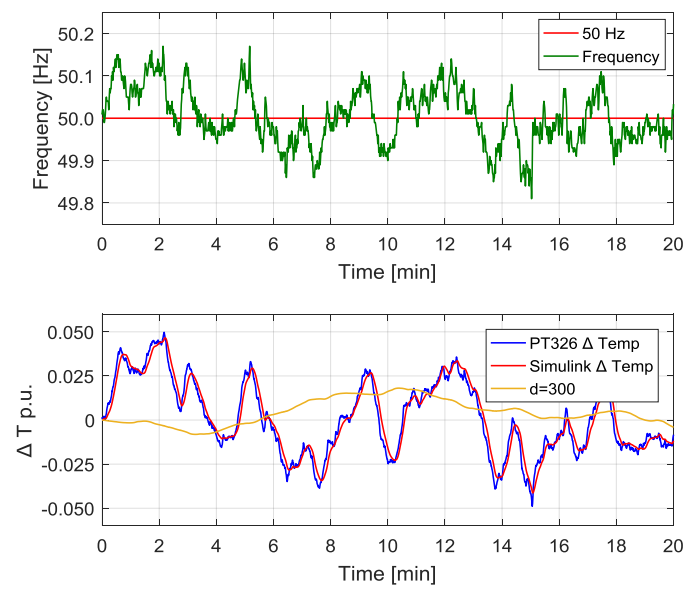

Figure 9 Frequency contribution to controllable load (TCL) 
Finally, Figure 9 [dataset] [60] illustrates the temperature recorded during simulated and HIL tests when mains frequency data was used as a time-series input signal to a controlled TCL. It shows a positive correlation between frequency and recorded temperature values with minimal delay. Although this is not fully representative of a practical closed-loop temperature control system in a building (where transport delay is typically much greater), it gives a good initial empirical demonstration that a thermal load can be driven in proportion to frequency measurement to quickly leverage a response in electrical demand. For completeness, a simulated TCL with a transport delay $d=300 \mathrm{~s}$ is also shown. During the same period, the variance in recorded temperature of a TCL with a greater transport delay was much less, due to the need to de-tune the closed-loop controller. Although predictive thermal controls are now routinely employed in many buildings, further investigation into the effects of the delay when under standard feedback control is an area of ongoing work.

There are important takeaways from the frequency analysis and effects of decentralised frequency control regulation tests. It reveals the measured data pertaining to the microcontroller is consistent with the first hypothesis regarding accuracy of frequency measurement. This allows dispatch of the frequency measurement instrumentation in a decentralised environment. Secondly, introduction of a small gain primary frequency regulator has a profound and positive influence on frequency restoration with no substantive change to measured temperature.

\section{Conclusions}

A decentralised frequency control regulation method has been validated using a series of computer models containing a single-area power network, a frequency measurement device and a real controllable thermal load (TCL). A FOPDT t.f. model of the TCL was initially identified and later substituted for a PT326 Process Trainer during HIL simulation tests. The overall approach was validated by controlling the temperature evolution (and electrical load) of the trainer directly from measured mains frequency data, by using it as an input signal to the closed-loop controllable load (TCL).

Design of a low-cost microcomputer frequency sensor and control element was discussed, and hypothesis tests provided evidence that substantiate claims that data collected using the Arduino frequency sensor is as good as data accessible from the National Grid. Further analysis has shown the action of the DFC-Primary regulator not only eliminates the observed frequency oscillation but also reduces the measured frequency deviation. Moreover, computer simulations have shown the contribution of the proposed DFC-Primary regulation action can potentially have a positive influence on reserve generation capacity, and provide an improved proactive alternative to demand response instruments [61]. The configuration of the regulator - including setting sensitivity to achieve a specific level of droop for a given heat pump, defining a deadband, etc. requires further study.

Several interesting conclusions can be drawn from the results presented. They suggest that small excursions in measured temperature from TCL setpoint values will not compromise indoor comfort temperatures but are capable of providing a contribution to the restoration of frequency equilibrium during network stress events. This means that the utility of a proactive decentralised control strategy directly could close the gap in reserve capacity margins availability by exploiting coupling technologies such as heat pumps and other TCLs with near zero intervention from the consumer. Another aspect within the energy market is the current trend to promote the number of active energy consumers, often called prosumers [62,63]. All the benefits discussed above support the various prosumer groups, the so called commercial prosumers; citizen led energy cooperatives and more generally single owners of build stock like universities, prisons and or public heath institutions.

In the wider context, it is likely that current technological and regulatory constraints, and societal perceptions of the smart-grid may motivate the utility of decentralised proactive frequency control of TCL to those that already benefit from a BEM system; the more popular systems using hysteresis control algorithm [64]. Coupling existing BEM technologies and the solution offered will not only improve the efficiency of local heat pumps (TCL) but also the whole building performance. This initiative will allow people to modify energy behaviour practices and participate in DR in such a way that is transparent. However, to dispatch effective proactive decentralised frequency control within existing energy markets, additional understanding is required. As a next step, computer models that analyse combinatorial problems usually associated with BEM, with the benefits that proactive decentralised frequency control offer will be undertaken. A natural progression of this work is to investigate the use of dynamic programming for optimal operation of building stock (including energy storage systems, TCL and access to real time data and dynamic pricing programs) to evaluate the benefits of the proposed concept in terms of grid economics and building performance.

In conclusion, the research presented in this paper suggests that frequency regulation of thermal loads in building stock provides a promising avenue for an advanced fast-acting balancing-service mechanism (decentralised demand response) to support smart energy networks. The proactive approach presented offers a viable alternative to more traditional system-balancing services that tend to be reactive (on/off) at set threshold values. This observation suggests the feasibility of using decentralised frequency control regulation as a novel approach to existing demand response mechanisms. Moreover, because of the propensity of EU Directives to characterise more energy efficient building construction this study may have important implications providing evidence in policymaking. 


\section{Acknowledgements}

The first author wishes to acknowledge the financial support provided by Teesside University and the Doctoral Training Alliance (DTA) scheme in Energy. The authors also acknowledge elements of the work was carried out as part of the DR-BOB project (01/03/16-28/02/19) which is co-funded by the EU's Horizon 2020 framework programme for research and innovation under grant agreement No 696114

\section{References}

[1] P. Mallet, P.-O. Granstrom, P. Hallberg, G. Lorenz, P. Mandatova, Power to the People!: European Perspectives on the Future of Electric Distribution, IEEE Power Energy Mag. (2014). doi:10.1109/MPE.2013.2294512.

[2] A. Snow, The first National Grid, Eng. Sci. Educ. J. 2 (1993) 215-224. doi:10.1049/esej:19930064.

[3] M. Lehtonen, S. Nye, History of electricity network control and distributed generation in the UK and Western Denmark, in: Energy Policy, IEEE, 2009: pp. 2338-2345. doi:10.1016/j.enpol.2009.01.026.

[4] M. Cheng, J. Wu, S.J. Galsworthy, N. Gargov, W.H. Hung, Y. Zhou, Performance of industrial melting pots in the provision of dynamic frequency response in the Great Britain power system, (2017). doi:10.1016/j.apenergy.2016.12.014.

[5] J. Villar, R. Bessa, M. Matos, Flexibility products and markets: Literature review, Electr. Power Syst. Res. 154 (2018) 329-340. doi:10.1016/j.epsr.2017.09.005.

[6] S. Matthewman, H. Byrd, Blackouts: a sociology of electrical power failure, Soc. Sp. (Przestrzeń Społeczna). 6 (n.d.) 31-55. https://issuu.com/socialspacejournal/docs/social_space_journal_22013_6_.

[7] Australia news, Electricity market operator denies being "asleep at the wheel" during blackout | Australia news | The Guardian, (n.d.). https:/www.theguardian.com/australia-news/2017/feb/10/electricity-marketoperator-denies-being-asleep-at-the-wheel-during-blackout (accessed October 15, 2017).

[8] M. Mureddu, G. Caldarelli, A. Chessa, A. Scala, A. Damiano, Green power grids: How energy from renewable sources affects networks and markets, PLoS One. (2015). doi:10.1371/journal.pone.0135312.

[9] A. Ulbig, T.S. Borsche, G. Andersson, Impact of low rotational inertia on power system stability and operation, in: IFAC Proc. Vol., 2014. doi:10.3182/20140824-6-ZA-1003.02615.

[10] R.F. Yan, T.K. Saha, N. Modi, N.A. Masood, M. Mosadeghy, The combined effects of high penetration of wind and PV on power system frequency response, Appl. Energy. (2015). doi:10.1016/j.apenergy.2015.02.044.

[11] C. Seneviratne, C. Ozansoy, Frequency response due to a large generator loss with the increasing penetration of wind/PV generation - A literature review, Renew. Sustain. Energy Rev. (2016). doi:10.1016/j.rser.2015.12.051.

[12] N. Jenkins, C. Long, J. Wu, An Overview of the Smart Grid in Great Britain, Engineering. 1 (2015) $413-421$. doi:10.15302/J-ENG-2015112.

[13] N. Good, K.A. Ellis, P. Mancarella, Review and classification of barriers and enablers of demand response in the smart grid, Renew. Sustain. Energy Rev. 72 (2017) 57-72. doi:10.1016/j.rser.2017.01.043.

[14] N.G. Paterakis, O. Erdinç, J.P.S. Catalão, An overview of Demand Response: Key-elements and international experience, Renew. Sustain. Energy Rev. 69 (2017) 871-891. doi:10.1016/j.rser.2016.11.167.

[15] R. Smale, B. van Vliet, G. Spaargaren, When social practices meet smart grids: Flexibility, grid management, and domestic consumption in The Netherlands, Energy Res. Soc. Sci. 34 (2017) 132-140. doi:10.1016/j.erss.2017.06.037.

[16] M.H. Shoreh, P. Siano, M. Shafie-khah, V. Loia, J.P.S. Catalão, A survey of industrial applications of Demand Response, Electr. Power Syst. Res. 141 (2016) 31-49. doi:10.1016/j.epsr.2016.07.008.

[17] Poyry, Costs and Benefits of GB Interconnection, 2016. https://www.nic.org.uk/wp-content/uploads/Costsand-benefits-of-GB-Interconnection-A-Poyry-Report.pdf.

[18] N. Richardson, M.J. Alexander, P. James, Energy storage against interconnection as a balancing mechanism for a 100\% renewable UK electricity grid, IET Renew. Power Gener. (2015). doi:10.1049/iet-rpg.2014.0042.

[19] D. Elliott, A balancing act for renewables, Nat. Energy. 1 (2016). http://dx.doi.org/10.1038/nenergy.2015.3.

[20] M.R. Sarker, M.D. Murbach, D.T. Schwartz, M.A. Ortega-Vazquez, Optimal operation of a battery energy storage system: Trade-off between grid economics and storage health, Electr. Power Syst. Res. (2017). 
doi:10.1016/j.epsr.2017.07.007.

[21] A. Aktas, K. Erhan, S. Ozdemir, E. Ozdemir, Experimental investigation of a new smart energy management algorithm for a hybrid energy storage system in smart grid applications, Electr. Power Syst. Res. 144 (2017). doi:10.1016/j.epsr.2016.11.022.

[22] T. Trainer, Some problems in storing renewable energy, Energy Policy. 110 (2017) 386-393. doi:10.1016/j.enpol.2017.07.061.

[23] V. Trovato, I.M. Sanz, B. Chaudhuri, G. Strbac, Advanced Control of Thermostatic Loads for Rapid Frequency Response in Great Britain, IEEE Trans. Power Syst. PP (2016) 1. doi:10.1109/TPWRS.2016.2604044.

[24] F. Conte, S. Massucco, F. Silvestro, Frequency control services by a building cooling system aggregate, Electr. Power Syst. Res. 141 (2016) 137-146. doi:10.1016/j.epsr.2016.07.003.

[25] F. Baccino, F. Conte, S. Massucco, F. Silvestro, S. Grillo, Frequency regulation by management of building cooling systems through Model Predictive Control, in: Proc. - 2014 Power Syst. Comput. Conf. PSCC 2014, 2014. doi:10.1109/PSCC.2014.7038380.

[26] F. Conte, S. Massucco, F. Silvestro, E. Ciapessoni, D. Cirio, Stochastic modelling of aggregated thermal loads for impact analysis of demand side frequency regulation in the case of Sardinia in 2020, Int. J. Electr. Power Energy Syst. 93 (2017) 291-307. doi:10.1016/j.ijepes.2017.05.030.

[27] M. Kamgarpour, C. Ellen, S.E.Z. Soudjani, S. Gerwinn, J.L. Mathieu, N. Mullner, A. Abate, D.S. Callaway, M. Franzle, J. Lygeros, Modeling options for demand side participation of thermostatically controlled loads, in: Proc. IREP Symp. Bulk Power Syst. Dyn. Control - IX Optim. Secur. Control Emerg. Power Grid, IREP 2013, 2013. doi:10.1109/IREP.2013.6629396.

[28] M. Marinelli, S. Martinenas, K. Knezović, P.B. Andersen, Validating a centralized approach to primary frequency control with series-produced electric vehicles, Adv. Life Course Res. 7 (2016) 63-73. doi:10.1016/j.est.2016.05.008.

[29] W. Wei, D. Wang, H. Jia, R. Wang, Y. Qi, M. Fan, X. Meng, A Communication Performance Evaluation on Smoothing Power Fluctuations Based on Demand Response Control of Thermostatically-controlled Appliances, in: Energy Procedia, 2015: pp. 1673-1678. doi:10.1016/j.egypro.2015.07.415.

[30] E. Kremers, J.M. González De Durana, O. Barambones, Emergent synchronisation properties of a refrigerator demand side management system, Appl. Energy. $101 \quad$ (2013) 709-717. doi:10.1016/j.apenergy.2012.07.021.

[31] D.S. Callaway, Tapping the energy storage potential in electric loads to deliver load following and regulation, with application to wind energy, Energy Convers. Manag. 50 (2009) 1389-1400. doi:10.1016/j.enconman.2008.12.012.

[32] Y.J. Kim, E. Fuentes, L.K. Norford, Experimental Study of Grid Frequency Regulation Ancillary Service of a Variable Speed Heat Pump, IEEE Trans. Power Syst. 31 (2016) 3090-3099. doi:10.1109/TPWRS.2015.2472497.

[33] F. Zhang, R. de Dear, C. Candido, Thermal comfort during temperature cycles induced by direct load control strategies of peak electricity demand management, Build. Environ. 103 (2016) 9-20. doi:http://dx.doi.org.ezproxy.tees.ac.uk/10.1016/j.buildenv.2016.03.020.

[34] T.S. Borsche, J. de Santiago, G. Andersson, Stochastic control of cooling appliances under disturbances for primary frequency reserves, Sustain. Energy, Grids Networks. $7 \quad$ (2016) 70-79. doi:http://dx.doi.org/10.1016/j.segan.2016.06.001.

[35] C.-J. Dalgaard, H. Strulik, Energy distribution and economic growth, Resour. Energy Econ. 33 (2011) $782-$ 797. doi:10.1016/j.reseneeco.2011.04.004.

[36] International Energy Agency, World Energy Outlook 2016, 2017. https://www.iea.org/publications/freepublications/publication/WorldEnergyOutlook2016ExecutiveSummary English.pdf.

[37] S. Verbeke, A. Audenaert, Thermal inertia in buildings: A review of impacts across climate and building use, Renew. Sustain. Energy Rev. (2017). doi:10.1016/j.rser.2017.08.083.

[38] A. Eichler, G. Darivianakis, J. Lygeros, Humans in the Loop: A Stochastic Predictive Approach to Building Energy Management in the Presence of Unpredictable Users, IFAC Proc. Vol. 50 (2017) 15036-15041. doi:10.1016/j.ifacol.2017.08.2295. 
[39] A. Reilly, O. Kinnane, The impact of thermal mass on building energy consumption, Appl. Energy. (2017). doi:10.1016/j.apenergy.2017.04.024.

[40] E.M.G. Rodrigues, R. Godina, E. Pouresmaeil, J.R. Ferreira, J.P.S. Catalão, Domestic appliances energy optimization with model predictive control, Energy Convers. Manag. (2017). doi:10.1016/j.enconman.2017.03.061.

[41] M. Dreidy, H. Mokhlis, S. Mekhilef, Inertia response and frequency control techniques for renewable energy sources: A review, Renew. Sustain. Energy Rev. (2017). doi:10.1016/j.rser.2016.11.170.

[42] A.M. Adil, Y. Ko, Socio-technical evolution of Decentralized Energy Systems: A critical review and implications for urban planning and policy, Renew. Sustain. Energy Rev. 57 (2016) 1025-1037. doi:10.1016/j.rser.2015.12.079.

[43] D. Fischer, H. Madani, On heat pumps in smart grids: A review, Renew. Sustain. Energy Rev. 70 (2017) 342-357. doi:10.1016/j.rser.2016.11.182.

[44] C. Wemhoener, R. Schwarz, L. Rominger, IEA HPT Annex 49-Design and integration of heat pumps in nZEB, Energy Procedia. 122 (2017) 661-666. doi:10.1016/J.EGYPRO.2017.07.366.

[45] V. Bianco, F. Scarpa, L.A. Tagliafico, Estimation of primary energy savings by using heat pumps for heating purposes in the residential sector, Appl. Therm. Eng. 114 (2017) 938-947. doi:10.1016/j.applthermaleng.2016.12.058.

[46] T. Crosbie, M. Short, M. Dawood, R. Charlesworth, Demand Response in Blocks of Buildings: Opportunities and Requirements, J. Entrep. Sustain. Issues. 4 (2017) 271-281.

[47] S. Williams, M. Short, T. Crosbie, Evaluating the role of building thermal inertia for the provision of decentralised demand side primary electrical frequency regulation services, in: SusTEM2017, 2017.

[48] H. Bevrani, T. Hiyama, Robust decentralised PI based LFC design for time delay power systems, Energy Convers. Manag. 49 (2008) 193-204. doi:http://dx.doi.org/10.1016/j.enconman.2007.06.021.

[49] Q. Shi, F. Li, Q. Hu, Z. Wang, Dynamic demand control for system frequency regulation: Concept review, algorithm comparison, and future vision, Electr. Power Syst. Res. 154 (2018) 75-87. doi:10.1016/j.epsr.2017.07.021.

[50] R. Sharma, G. Dhole, Least Error Squares Approach: A Practical Method for Power System Frequency and Amplitude Estimation, Procedia Technol. 25 (2016) 710-717. doi:10.1016/J.PROTCY.2016.08.164.

[51] Atmel Corporation, Atmel ATmega2560 Datasheet, (2014). http://www.atmel.com/Images/Atmel-2549-8bit-AVR-Microcontroller-ATmega640-1280-1281-2560-2561_datasheet.pdf (accessed October 6, 2017).

[52] MathWorks, MATLAB R2016b and Simulink, (2016).

[53] S. Nag, N. Philip, Application of neural networks to automatic load frequency control, in: Control. Instrumentation, Energy Commun. (CIEC), 2014 Int. Conf., 2014: pp. 345-350. doi:10.1109/CIEC.2014.6959107.

[54] S. Wang, R. Tang, Supply-based feedback control strategy of air-conditioning systems for direct load control of buildings responding to urgent requests of smart grids, Appl. Energy. (n.d.). doi:http://dx.doi.org/10.1016/j.apenergy.2016.10.067.

[55] E.. Mathews, P.. Richards, C. Lombard, A first-order thermal model for building design, Energy Build. 21 (1994) 133-145.

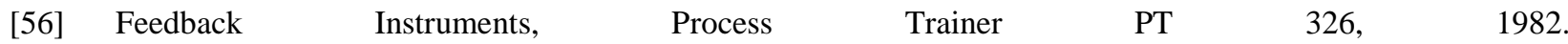
https://eclass.upatras.gr/modules/document/file.php/EE817/thermic_PT_326_manual.pdf.

[57] T. (Emerson) Olsen, B. (Emerson) Bialkowski, Lambda Tuning as a Promising Controller Tuning Method for the Refinery, AIChE Spring Natl. Meet. (2002).

[58] D.E. Rlvera, M. Morarl, S. Skogestad, Internal Model Control. 4. P I D Controller Design, Ind. Eng. Chem. Process Des. Dev. 25 (1986) 252-265. http://pubs.acs.org/doi/pdf/10.1021/i200032a041 (accessed October 6, 2017).

[59] ELEXON Limited, Balancing Mechanism Reporting Service, (n.d.). https://www.bmreports.com/bmrs/?q=help/about-us (accessed February 12, 2017).

[60] S. Williams, ATE_2017_6249 Mendeley Data, v1, (2017). doi:http://dx.doi.org/10.17632/5kw6y64x52.1.

[61] A. Molina-Garcia, F. Bouffard, D.S. Kirschen, Decentralized Demand-Side Contribution to Primary 
Frequency Control, IEEE Trans. Power Syst. 26 (2011) 411-419. doi:10.1109/TPWRS.2010.2048223.

[62] W. Miller, M. Senadeera, Social transition from energy consumers to prosumers: Rethinking the purpose and functionality of eco-feedback technologies, Sustain. Cities Soc. 35 (2017) 615-625. doi:10.1016/j.scs.2017.09.009.

[63] K. Buchanan, R. Russo, B. Anderson, Feeding back about eco-feedback: How do consumers use and respond to energy monitors?, Energy Policy. 73 (2014) 138-146. doi:10.1016/j.enpol.2014.05.008.

[64] D.W.U. Perera, D. Winkler, N.-O. Skeie, Multi-floor building heating models in MATLAB and Modelica

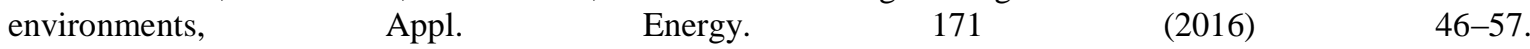
doi:http://dx.doi.org.ezproxy.tees.ac.uk/10.1016/j.apenergy.2016.02.143.

\section{Appendix 1}

MATLAB/Simulink@ model parameters:

Power system rating: $300 \mathrm{MVA}$

Nominal frequency $(f): 50 \mathrm{~Hz}$

Nominal demand load $(P d): 75 \mathrm{MW}$

ALFC secondary loop gain $(K i): 0.2$ p.u. MW/Hz s

Speed regulator $(R): 0.05 \mathrm{~Hz} /$ p.u. MW

Inertia time constant $(H): 5 \mathrm{~s}$

Load damping constant $(D): 0.8 \mathrm{~s}$

Governor time constant $\left(T_{g}\right): 0.25 \mathrm{~s}$

Turbine time constant $\left(T_{t}\right): 0.60 \mathrm{~s}$

DFC-Primary regulator $(R): 0.05$

Thermal load time constant $\left(T_{h}\right): 9.65 \mathrm{~s}$

FOPDT thermal load gain $\left(K_{h}\right): 1.16$

FOPDT transport delay $(d): 0.45$

TCL controller proportional gain $(P): 0.51750$

TCL controller integral gain $(I): 0.10363$

Temperature setpoint $(O F 1): 7.5\left(\equiv 40^{\circ} \mathrm{C}\right)$

Secondary Demand Response gain $(S D R): 0.5$

Calibration factor (electrical power) (OF2): 0.274

Calibration factor (temperature) (OF3): 0.345

Compensator gain $(G 1): 20$

Compensator gain $(G 2, G 3): 1 / G 1$

\section{Appendix 2}




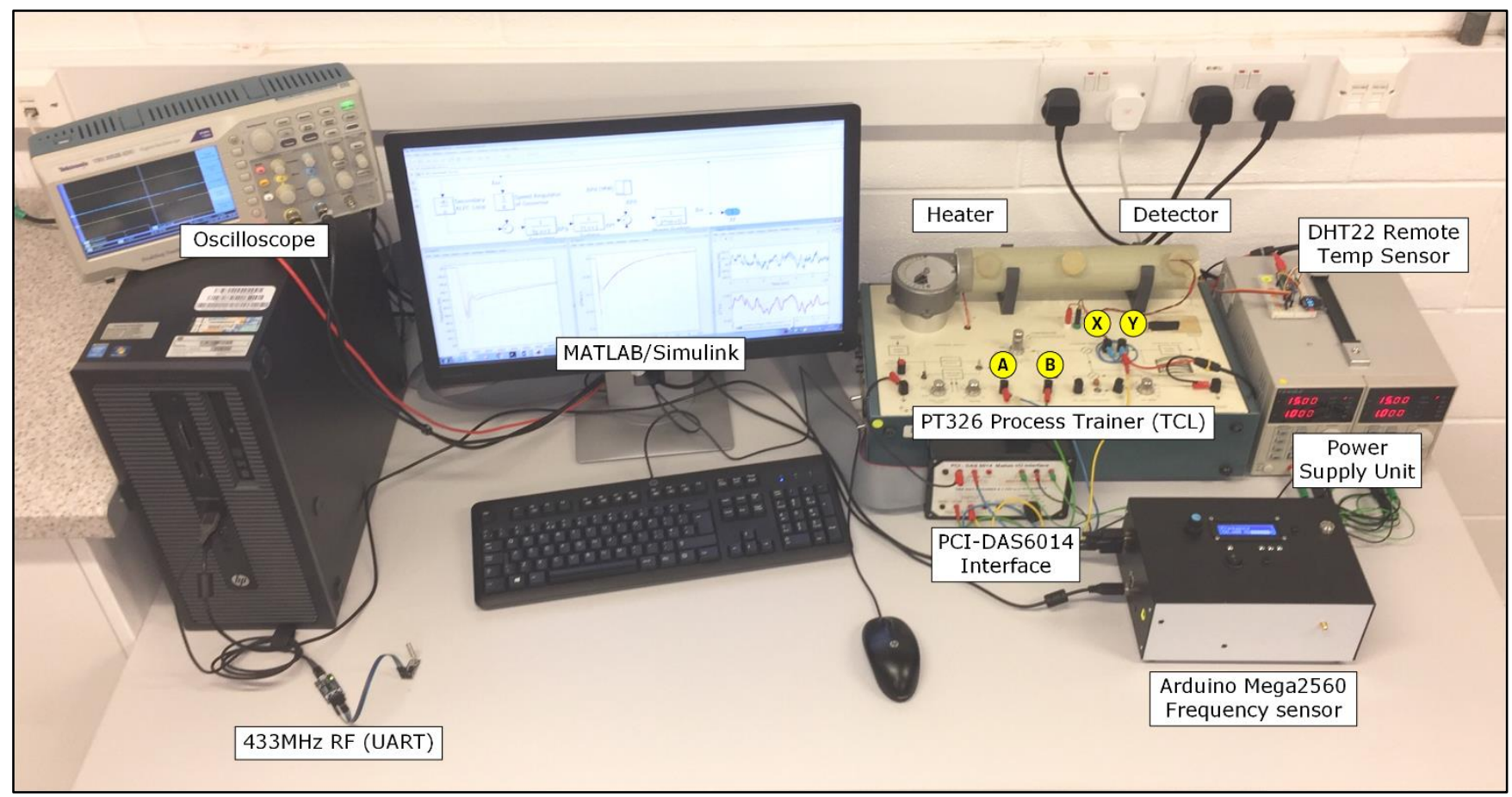

Figure 10 HIL simulation test environment 